\title{
Diagnostic, pronostic et traitements des troubles de la conscience
}

\section{Diagnosis, prognosis and treatment in disorders of consciousness}

\author{
H. Cassol ${ }^{*}$, C. Aubinet, A. Thibaut ${ }^{1}$, S. Wannez, \\ C. Martial, G. Martens, S. Laureys
}

Coma Science Group, GIGA-Consciousness and Neurology Department, University and University Hospital of Liège, Liège, Belgique

Disponible sur Internet le 3 juin 2017

\section{MOTS CLÉS}

Conscience ;

Lésion cérébrale ;

Diagnostic ;

Pronostic ;

Traitement ;

États de conscience

altérée ;

Coma ;

État végétatif ;
Résumé Les progrès de la médecine et des soins intensifs ont conduit à une augmentation du nombre de patients survivant à une lésion cérébrale sévère. Bien que certains patients récupèrent rapidement, d'autres demeurent dans un état de conscience altérée (ECA). Ces derniers peuvent évoluer du coma vers un état végétatif/syndrome d'éveil non répondant (EV/ENR), puis vers un état de conscience minimale $(E C M)$. Dans cette revue, nous proposons tout d'abord de décrire les différentes méthodes, comportementales et de neuro-imagerie, utilisées dans le diagnostic des patients en ECA. Nous décrirons ensuite les facteurs susceptibles d'influencer le pronostic et la récupération de ces patients, ainsi que les traitements et la prise en charge qui

\footnotetext{
* Auteur correspondant. GIGA (ULg) B34, 11, avenue de l'Hôpital, 4000 Liège, Belgique.

Adresse e-mail : hcassol@ulg.ac.be (H. Cassol).

${ }^{1}$ Aurore Thibaut a supervisé et guidé la rédaction de cette revue.
} 
Éveil non répondant ; État de conscience minimale ;

Locked-in syndrome ;

Neuro-imagerie ;

Tomographie par

émission de positons ;

Coma Recovery

Scale-Revised ;

Imagerie par

résonance

magnétique ;

Électroencéphalogra-

phie ;

Stimulation

magnétique

transcrânienne peuvent être proposés dans le but d'améliorer leur état de conscience. Enfin, nous clôturerons cette revue avec une réflexion sur les considérations éthiques et les questions de fin de vie.

(c) 2017 Elsevier Masson SAS. Tous droits réservés.

\section{KEYWORDS \\ Consciousness; \\ Brain injury; \\ Diagnosis; \\ Prognosis; \\ Treatment; \\ Altered states of \\ consciousness; \\ Coma; \\ Vegetative state; \\ Unresponsive \\ wakefulness \\ syndrome; \\ Minimally conscious \\ state; \\ Locked-in syndrome; \\ Neuroimaging; \\ positron emission \\ tomography; \\ Coma Recovery \\ Scale-Revised; \\ Magnetic resonance; \\ Electroencephalogra- \\ phy; \\ Transcranial \\ magnetic stimulation}

Summary Advances in medicine and intensive care have led to an increase in the number of patients surviving a severe brain injury. Although some patients recover quickly, others remain in a state of altered consciousness. These patients can progress from a coma to a vegetative state or unresponsive wakefulness syndrome (VS/UWS) and later to a minimally conscious state (MCS). In this review, we first describe the different behavioral and neuroimaging methods used in the diagnosis of patients in an altered state of consciousness. We then describe the factors that can influence the prognosis and recovery of these patients, as well as the treatment and therapeutic management that can be proposed in order to improve their state of consciousness. Finally, we conclude this review by highlighting ethical considerations and end-of-life issues. (c) 2017 Elsevier Masson SAS. All rights reserved.

\section{Introduction les états de conscience altérées $(E C A)$-définitions}

La conscience de l'individu réfère à l'émergence simultanée de deux composantes particulières (Fig. 1) :

- le niveau d'éveil ;

- le contenu de la conscience, qui lui-même se caractérise par deux sous-composantes, la conscience de soi (conscience interne) et de son environnement (conscience externe) $[1,2]$.
L'éveil, ou la vigilance, est principalement caractérisé par une ouverture des yeux alors que le contenu de la conscience est cliniquement objectivé par la présence d'interactions avec l'environnement et de réponses à la commande. Ces composantes sont évidentes chez un individu éveillé et pleinement conscient mais elles décroissent lorsqu'il s'endort : plus il est endormi, moins il est à la fois vigilant et réceptif [1]. Lorsque des patients sont anesthésiés, ces deux composantes de conscience sont alors absentes ou minimales [3]. Le patient récupère ensuite un 


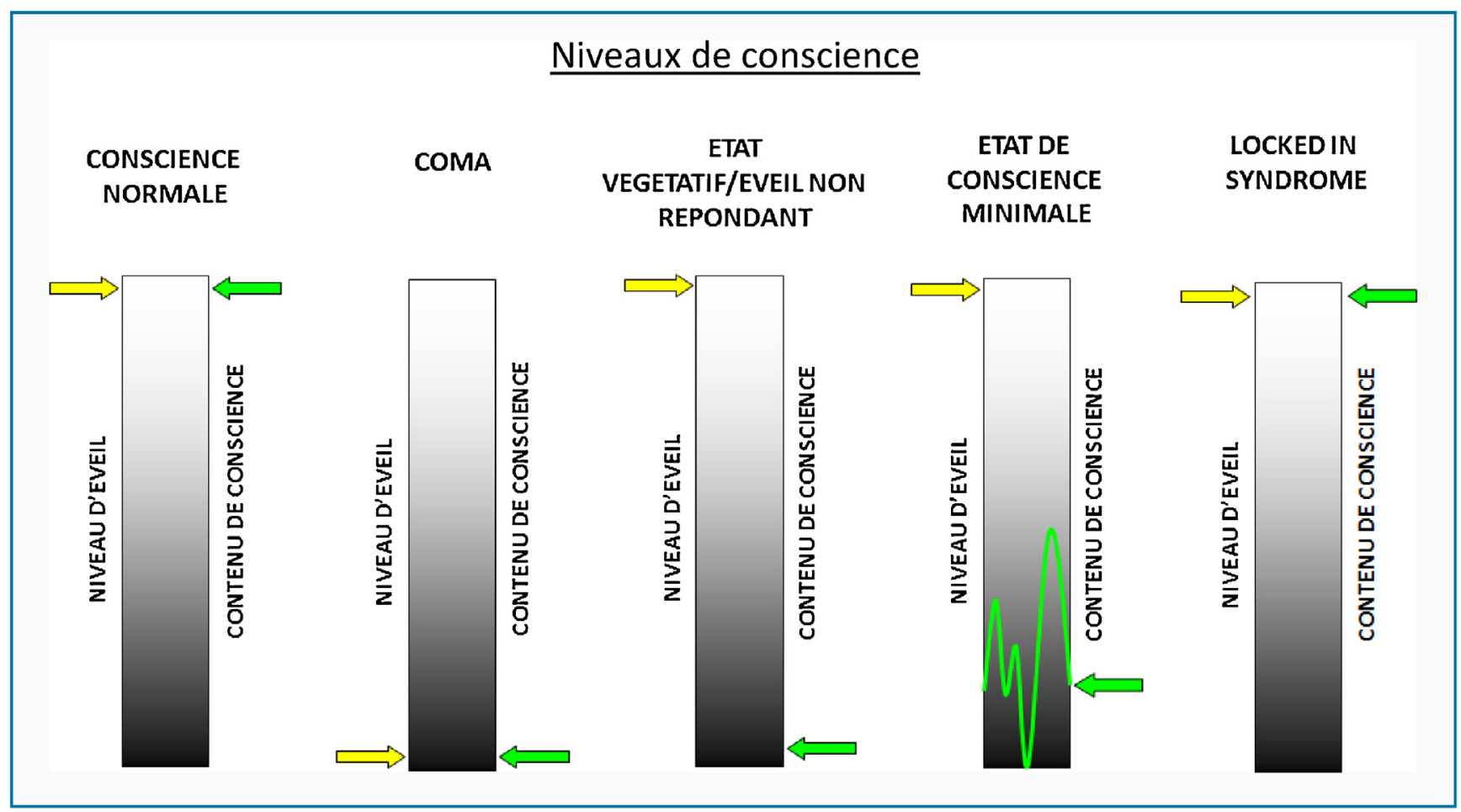

Figure 1. Représentation du niveau d'éveil et du contenu de conscience pour les différents états de conscience (adapté de [5]).

niveau de conscience normal. Les deux composantes de la conscience sont également absentes chez des patients dans le coma ; ils n'ouvrent pas les yeux et ne sont conscients ni d'eux-mêmes, ni de leur environnement. Ces patients peuvent récupérer complètement ou passer par différents stades que l'on nomme « états de conscience altérée » (ECA).

Les patients en ECA ont été particulièrement étudiés durant les dernières décennies [4]. Après un coma, ils peuvent récupérer complètement, évoluer vers une mort cérébrale ou vers un état végétatif dit d' « éveil non répondant » (EV/ENR) $[5,6]$.

La mort cérébrale (ou mort encéphalique) est définie par les critères suivants :

- la présence d'un coma profond et irréversible ;

- l'exclusion de facteurs susceptibles d'entraîner un tableau clinique identique, mais réversible, tels que l'hypothermie ;

- l'abolition de toutes les fonctions du tronc cérébral ;

- l'absence de réponses motrices ;

- l'apnée. Une évaluation répétée à 6 heures (période considérée comme arbitraire) d'intervalle est conseillée. Les tests de laboratoire confirmatoires ne sont nécessaires que lorsque des composantes spécifiques des tests cliniques ne peuvent être évaluées de manière fiable [7].

L'EV/ENR est quant à lui caractérisé par une importante dissociation entre les composantes d'éveil et de contenu de conscience. En effet, dans cet état pathologique, les patients présentent un haut niveau d'éveil (ouverture des yeux spontanée ou à la suite d'une stimulation auditive, tactile ou nociceptive), mais aucune interaction avec leur environnement ni aucun comportement volontaire [8].
Lorsqu'ils récupèrent davantage, certains patients peuvent montrer des signes de conscience tels que la poursuite visuelle, une manipulation d'objets ou une localisation de la douleur, et ce de façon reproductible mais également fluctuante (un signe peut être observé le matin et être absent lorsque le patient est réévalué quelques heures plus tard). Ces patients sont dits en «état de conscience minimale » (ECM) [9]. Ce trouble de la conscience a été récemment divisé en ECM plus et ECM moins, respectivement selon la présence ou l'absence de réponses à la commande (récupération du réseau du langage-[10]). Enfin, un patient émerge de l'ECM lorsqu'il montre une communication fonctionnelle (verbale ou non) ou une utilisation fonctionnelle d'objets [9]. Le patient n'est dès lors plus considéré comme étant en ECA. Notons qu'un diagnostic différentiel doit également être établi par rapport au syndrome «lockedin », qui concerne des patients pleinement conscients, mais dépourvus de contrôle moteur, typiquement à l'exception de mouvements oculaires.

Plusieurs types d'étiologies peuvent mener les patients à un EV/ENR ou à un ECM. Selon Leonardi et al. [11], une majorité d'entre eux a subi un accident non traumatique. En effet, parmi leur échantillon de 564 patients adultes, 171 ont subi une hypoxie, 165 une hémorragie cérébrale et 38 un infarctus cérébral, contre 148 patients présentant une étiologie traumatique. L'âge des patients influence cette étiologie, les individus âgés étant davantage exposés aux risques cardiovasculaires, pouvant mener à un accident vasculaire cérébral ou à une hypoxie cérébrale post-arrêt cardiaque, alors que les patients plus jeunes sont plus à risque d'être victime d'un accident traumatique.

Les états pathologiques de conscience altérée peuvent donc survenir à tout âge et renvoient à des situations 
familiales, humaines et éthiques particulièrement dramatiques. Grâce à l'étude de ces ECA, des découvertes sur les réseaux de la conscience ont pu émerger. Par exemple, le niveau de conscience a longtemps été défini comme une propriété émergente de l'activité cérébrale en général. Il a pourtant été démontré que lorsque des patients en ECA recouvrent un niveau de conscience normale, celle-ci n'est pas accompagnée d'une augmentation significative du métabolisme cérébral global [5]. Cette découverte a constitué un premier indice indiquant que l'émergence de la conscience serait sous-tendue par un large réseau comprenant des régions corticales spécifiques.

Le premier objectif de cette revue de la littérature est de préciser les différentes méthodes d'évaluation des ECA, combinant des échelles comportementales et diverses techniques de neuro-imagerie. Notre second objectif consiste ensuite à faire le point sur le pronostic qu'imposent ces ECA. Enfin, nous discuterons de la prise en charge des patients en ECA, que ce soit au niveau pharmacologique ou de la revalidation clinique.

\section{Outils diagnostiques}

\section{Échelles comportementales}

Les évaluations comportementales sont le premier outil que le clinicien peut utiliser lorsqu'il s'agit de diagnostiquer un patient en ECA. Il est important d'utiliser une échelle standardisée, sensible et validée, car un diagnostic posé sans se servir de tels outils peut mener à un haut taux d'erreurs diagnostiques $[12,13]$. Ce diagnostic est essentiel puisqu'il va influencer la prise en charge des patients en ECA et peut donc avoir un impact important sur leur pronostic.

L'échelle clinique la plus connue et utilisée est sans conteste la Glasgow Coma Scale [14]. Si celle-ci est particulièrement utile pour évaluer un patient en phase aiguë, elle n'est toutefois pas la mieux adaptée pour différencier les patients post-coma qui s'éveillent et entrent progressivement en EV/ENR ou en ECM [15].

Selon de récentes recommandations [16], l'échelle comportementale la plus adaptée et la plus sensible est la Coma Recovery Scale-Revised (CRS-R) [17], composée de 23 items hiérarchisés, du réflexe aux comportement cognitifs plus élevés, répartis dans différentes sous-échelles : auditive, visuelle, motrice, oromotrice/verbale, communication et éveil. Alors que les items les plus bas représentent des réflexes, les plus hauts indiquent la présence d'un ECM ou de l'émergence de l'ECM. La CRS-R permet de calculer un score total entre 0 et 23 , en additionnant les meilleurs scores de chaque sous-échelle. Le score total peut être utile pour quantifier l'évolution d'un patient. Récemment, une étude a montré que des patients inconscients et conscients peuvent être diagnostiqués correctement dans $94 \%$ des cas si un score seuil de 8 est utilisé [18]. Cependant, le score total ne peut pas être utilisé comme seul outil diagnostique. L'analyse qualitative des comportements montrés par le patient reste donc indispensable pour poser un diagnostic correct. Certaines sous-échelles semblent plus sensibles que d'autres pour détecter un signe de conscience et donc poser le diagnostic d'ECM. Ainsi, chez plus de $80 \%$ des patients en ECM, la sous-échelle visuelle détecte un signe de conscience $[19,20]$. Cette sous-échelle contient entre autres la poursuite visuelle, qui est observée chez 55 à $70 \%$ des patients en ECM et qui est un des premiers signes de conscience à être récupéré $[21,22]$. De plus, l'implémentation de cette échelle doit se faire avec des outils appropriés. Par exemple, la poursuite visuelle sera significativement plus souvent observée si elle est testée avec un miroir et le patient localisera davantage un son s'il est appelé par son prénom [21-24]. Une récente étude a également montré que certains items étaient plus fréquemment observés chez les patients en ECM : la réponse à la commande, la poursuite visuelle, la fixation, les comportements moteurs automatiques et la localisation à la douleur. Les cliniciens pourraient donc concentrer leurs efforts sur ces items lorsqu'ils manquent de temps ou que les patients sont trop fatigués [25].

Les résultats des évaluations comportementales peuvent dépendre de plusieurs paramètres comme l'expertise de l'évaluateur [26]. La durée de l'évaluation peut également influencer le diagnostic : étant donné que les patients peuvent fluctuer, une augmentation du temps passé auprès d'eux augmente également les chances de détecter un signe de conscience [27]. La présence de la famille peut également influencer le diagnostic [28]. Or, un diagnostic correct est important pour diverses raisons éthiques et médicales : d'une part, le pronostic du patient dépend non seulement de l'étiologie, mais aussi de ce diagnostic [29] ; d'autre part, il est susceptible d'influencer diverses décisions médicales telles que le traitement antalgique et une éventuelle fin de vie $[30,31]$. Notons que, bien que la CRS-R soit l'outil le plus sensible pour diagnostiquer les ECA, 32 \% des patients considérés comme inconscients comportementalement montrent une activité cérébrale compatible avec un ECM lorsqu'ils sont évalués avec des outils de neuro-imagerie [13]. Dans ce contexte, une approche multidisciplinaire apparaît essentielle afin de poser le diagnostic le plus précis possible.

\section{Neuro-imagerie}

Les techniques de neuro-imagerie permettent de comparer l'aspect structurel et fonctionnel du cerveau de patients avec celui de sujets sains afin de contribuer au jugement clinique, notamment lorsque les signes de conscience comportementaux sont insuffisants ou ambigus [32]. Certains patients peuvent en effet souffrir de lésions motrices qui les empêchent d'exprimer un signe comportemental de conscience, alors qu'ils sont cognitivement aptes à comprendre certaines commandes. En effet, la neuroimagerie apporte des informations quant aux atteintes cérébrales structurelles et fonctionnelles. Les réseaux fonctionnels résiduels peuvent être étudiés lorsque le cerveau est engagé dans une tâche (paradigme actif) ou lorsqu'il est au repos. L'inconvénient du paradigme actif est qu'il exige la préservation du langage et la collaboration du patient alors que la présence d'une aphasie de compréhension est probable chez de nombreux patients en ECA [33]. Les paradigmes passifs, quant à eux, ne nécessitent pas la participation active du patient. Les outils de neuro-imagerie sont toujours utilisés en complément de l'évaluation clinique et le diagnostic du patient se base sur l'entièreté des examens effectués. Ainsi, jusqu'à $30 \%$ des patients ne montrent cliniquement aucun signe de conscience, mais 
présentent une activité cérébrale compatible avec une conscience résiduelle [13]. Ces patients sont considérés en ECM non comportemental [23]. À l'inverse, si malgré les différents examens aucune activité cérébrale compatible avec une conscience résiduelle n'est détectée, cela exclut la possibilité d'un ECM non comportemental et plaide en faveur d'un ENR ou d'un coma (en fonction de si le patient ouvre les yeux ou non, respectivement). Les outils de neuro-imagerie sont utiles pour compléter le diagnostic clinique, mais ne peuvent s'y substituer [13].

\section{Tomographie par émission de positons (TEP)}

En mesurant le métabolisme et en évaluant le débit sanguin cérébral, la tomographie par émission de positons (TEP) permet de complémenter le diagnostic posé à l'aide d'échelles comportementales comme la CRS-R en détectant des signes de conscience. La TEP permet d'étudier le fonctionnement cérébral régional sans la participation active des patients, grâce à l'injection de radio-isotopes utilisés comme traceurs (Fig. 2). Les marqueurs ayant le plus souvent été utilisés dans l'exploration des altérations de la conscience sont le fluorodésoxyglucose $\left({ }^{18} \mathrm{FDG}\right)$, qui évalue le métabolisme cérébral au repos, et l'eau marquée à l'oxygène $15\left(\mathrm{H}_{2} \mathrm{O}^{15}\right)$, pour l'enregistrement en réponse à un stimulus spécifique (régions (dés)activées en réponse à un stimulus auditif, visuel ou nociceptif) [34].

Récemment, Stender et al. [35] ont comparé le métabolisme cérébral au repos de sujets témoins à celui de patients sévèrement cérébrolésés, et ont mis en évidence un déclin global du métabolisme cérébral chez ces derniers. L'activité métabolique globale était significativement plus élevée chez les patients en ECM (55\% de la normale) en comparaison avec les patients en EV/ENR (42\% de la normale). Cependant, il semble que la mesure du métabolisme global ne soit pas systématiquement représentative du niveau de conscience. En effet, certains sujets témoins présentent un métabolisme cérébral comparable aux patients EV/ENR [1], de surcroît, l'évolution favorable de l'état de conscience de ces derniers n'est pas toujours accompagnée d'une augmentation du métabolisme cérébral global. Ces résultats suggèrent que certaines régions corticales sont davantage impliquées dans l'émergence de la conscience $[1,36]$. En effet, les patients en EV/ENR montrent une activité réduite dans toutes les régions corticales avec, néanmoins, un déclin plus important au sein des cortex sensoriels et moteurs, du réseau frontopariétal et du thalamus [5,35]. Par ailleurs, ces patients présentent un métabolisme du tronc cérébral relativement préservé, ce qui concorde avec la préservation de leur niveau d'éveil et des fonctions autonomes telles que la respiration ou la thermorégulation [37]. Par ailleurs, la récupération de la conscience semble associée au rétablissement de l'activité cérébrale au sein d'un large réseau frontopariétal, ainsi qu'à la restauration des connexions fonctionnelles à longue distance au sein de ce réseau et entre certaines aires corticales associatives et le thalamus [36]. Plus précisément, le réseau frontopariétal est lui-même composé de deux réseaux distincts :

- le réseau du mode par défaut (le cortex cingulaire postérieur/précunéus, le cortex cingulaire antérieur/mésiofrontal et certaines zones de la jonction temporopariétale), impliqué dans les processus de perception interne et dans la conscience de soi ;

- le réseau externe et de contrôle exécutif (réseau frontopariétal latéral), quant à lui impliqué dans les processus de perception externe et dans la conscience de l'environnement [38].

\section{Imagerie par résonance magnétique (IRM) IRM structurelle}

Certaines études d'IRM ont été réalisées chez les patients en ECA afin d'estimer les dommages structurels de leur cerveau. Guldenmund et al. [2016] ont par exemple récemment utilisé la morphométrie basée sur les voxels (technique permettant d'évaluer l'intégrité structurelle de chaque voxel) et ont montré une corrélation positive entre l'atteinte structurelle et le niveau de conscience. En outre, une durée plus importante du trouble était associée à une atteinte structurelle plus étendue. Comparativement aux non traumatiques, les étiologies traumatiques étaient liées à des dommages plus importants au niveau du tronc cérébral, du mésencéphale, du thalamus, de l'hypothalamus, du prosencéphale basal, du cervelet et du corps calleux postérieur. Des différences structurelles ont également été mises en évidence entre l'EV/ENR et l'ECM, montrant une atteinte moins sévère du cortex préfrontal ventro-médial et du précuneus/cortex cingulaire postérieur chez les patients en ECM [39]. Une autre technique permettant d'objectiver l'atteinte des connections au niveau de la matière blanche est l'imagerie par tenseur de diffusion («diffusion weighted imaging »). L'importance des connections thalamocorticales au niveau du profil comportemental et du niveau de conscience des patients en ECA a ainsi dernièrement été mise en évidence [40].

\section{IRM fonctionnelle}

Lorsqu'aucune réponse à la commande ne peut être observée grâce aux échelles comportementales, à cause de lésions motrices extensives par exemple, un paradigme actif d'IRM peut permettre d'étudier ce comportement chez certains patients en ECA. Ce paradigme consiste à demander au patient de s'imaginer en train de jouer au tennis ou en train d'arpenter sa maison. Il a été choisi car il active des régions bien distinctes du cerveau. Dans le premier cas, les sujets témoins activent l'aire motrice supplémentaire; dans le second, il s'agit du cortex prémoteur latéral, du lobe pariétal postérieur et du gyrus parahippocampique [41]. Si un patient en ECA présente une activation dans ces régions cérébrales typiquement associées avec une de ces tâches, cela suggère que le patient a compris et exécuté la commande, et donc que son réseau du langage est préservé (Fig. 3). Il est alors important de prendre cela en compte lors de la prise en charge future du patient. Dans une étude utilisant ce paradigme [41], ces commandes ont été présentées oralement à 54 patients. Parmi ceux-ci, 5 ont montré une telle réponse, dont 2 étaient incapables de répondre à la commande en dehors du scanner. Cette étude a également prouvé qu'il est possible de tenter de communiquer via un code « oui/non» sur base de ce dispositif. En effet, celui-ci a permis à un patient de répondre « oui » à une question en pensant qu'il jouait au tennis et d'y répondre par «non » en s'imaginant arpenter sa maison. 


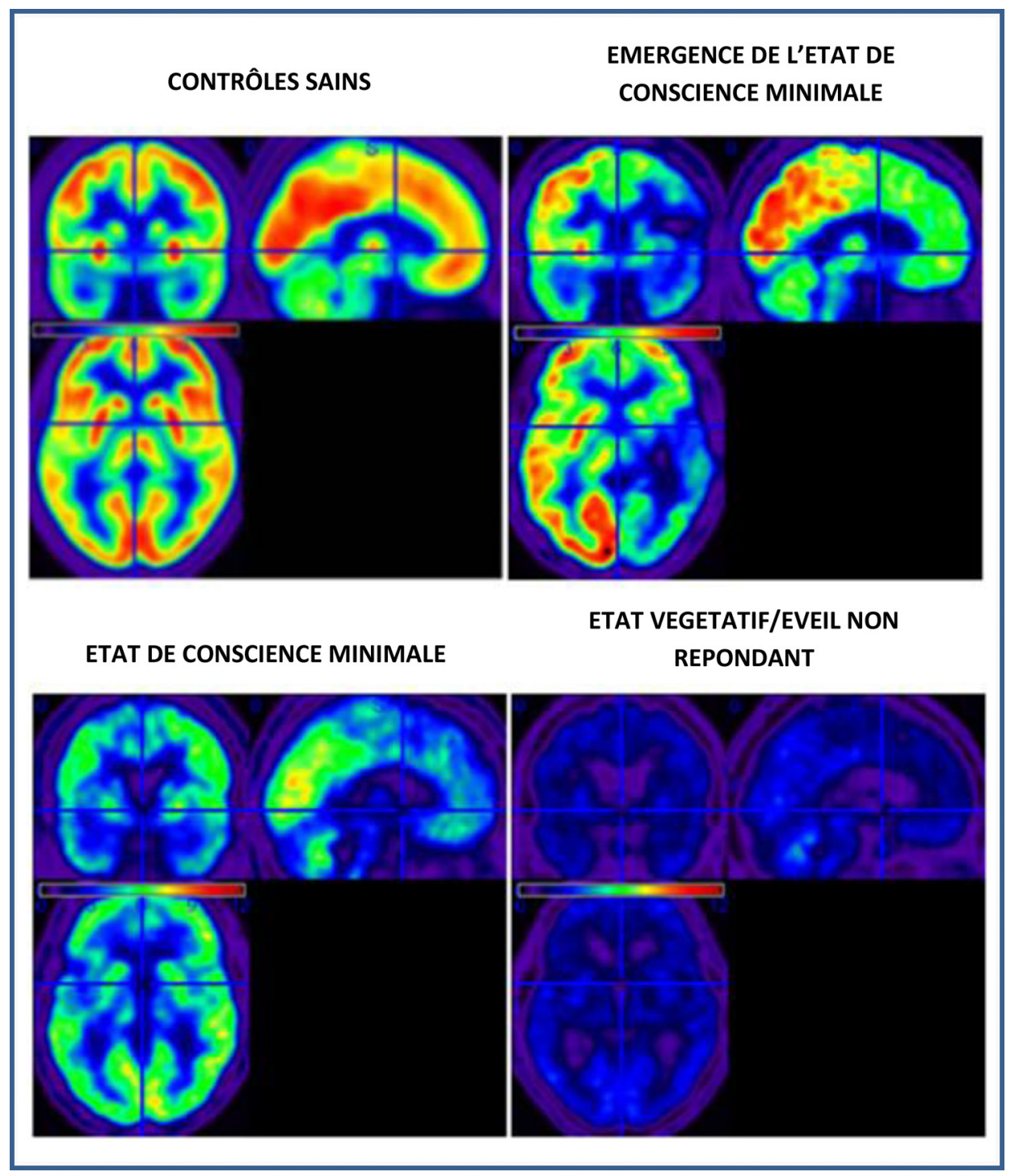

Figure 2. Métabolisme cérébral global chez des sujets témoins et chez des patients en état de conscience altérée mesuré à l'aide du ${ }^{18} \mathrm{FDG}$-TEP. Les régions en rouge indiquent une consommation en glucose importante, les régions en bleu indiquent une consommation en glucose faible.

Au vu de l'absence de collaboration des patients en ECA, certaines recherches d'IRM fonctionnelle se sont basées sur un paradigme actif événementiel. Par exemple, Schiff et al. [42] ont étudié les réponses corticales de patients en ECM lors de blocs de stimulations langagières et tactiles. Ils ont conclu que ces patients présentaient une activation des systèmes corticaux diffus susceptibles de soutenir certaines fonctions cognitives et sensorielles, et ce malgré leur incapacité à suivre des instructions simples ou à communiquer fonctionnellement. D'autres auteurs ont récemment investigué l'impact de la musique sur la connectivité fonctionnelle cérébrale chez des patients en ECA en comparant une condition « musique préférée » à une condition contrôle (bruit de l'IRM) [43]. À l'écoute de la musique préférée, la connectivité fonctionnelle était significativement plus importante au niveau du réseau auditif (impliqué dans la perception du rythme et de la musique), mais aussi au niveau de la jonction temporopariétale du réseau externe (en lien avec la mémoire autobiographique).
Le paradigme au repos, fondé sur les variations spontanées de la quantité d'oxygène dans le sang, a également été beaucoup utilisé dans ce champ de recherche. Plusieurs études ont notamment montré que la connectivité fonctionnelle du réseau du mode par défaut diminue plus le niveau de conscience est bas (conscient $>E C M>E V / E N R>$ coma) $[38,44,45]$.

\section{Électroencéphalographie (EEG)}

Bien que les techniques de neuro-imagerie TEP et IRM soient des outils très précieux dans la détection de signes de conscience, l'électroencéphalogramme (EEG) présente plusieurs avantages non négligeables dans cette démarche : il peut s'utiliser directement au chevet des patients, il est plus facilement accessible cliniquement et moins onéreux, et il offre une résolution temporelle plus élevée. Cette haute résolution temporelle (de l'ordre de la milliseconde) joue 


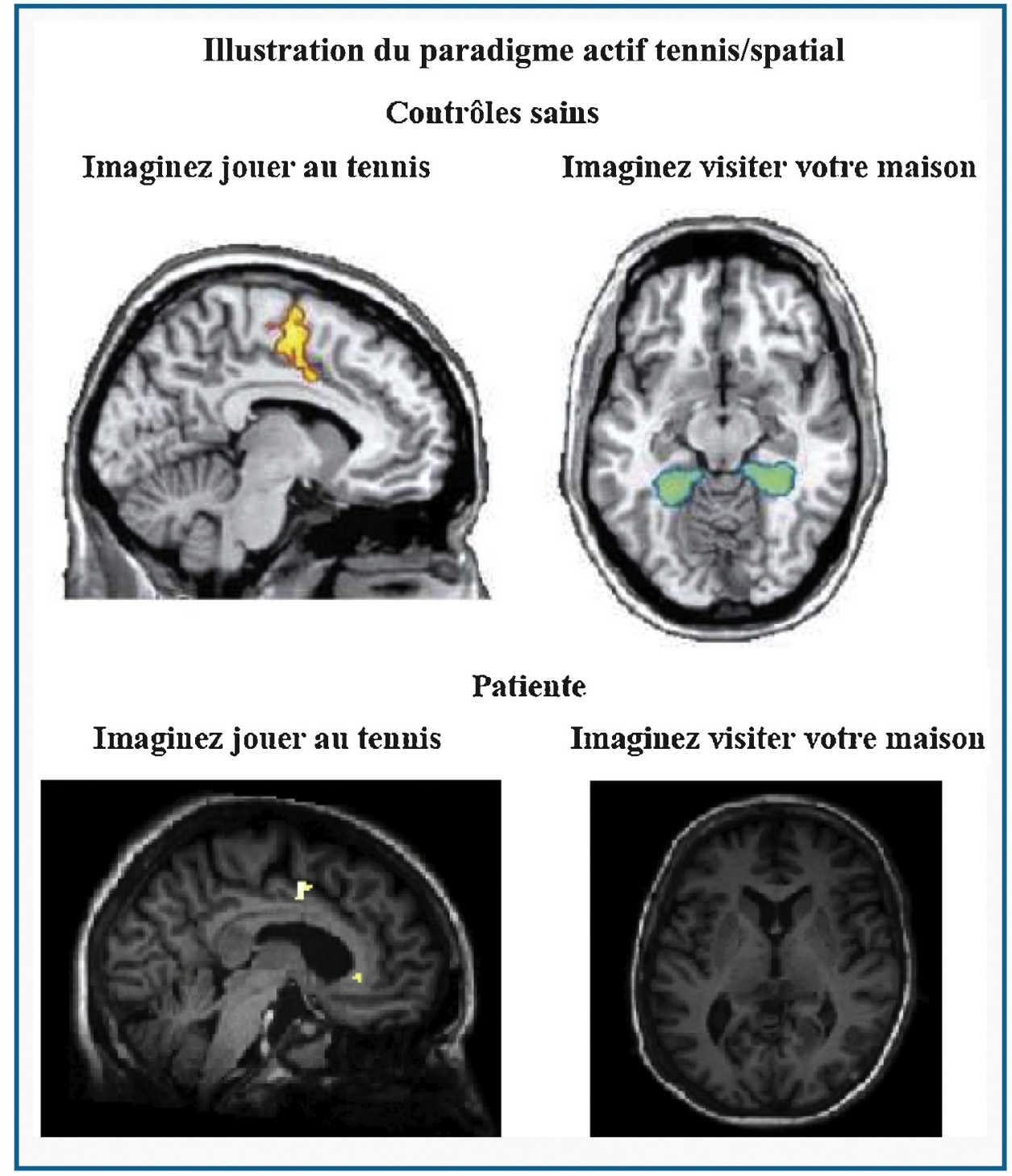

Figure 3. En haut : région activée-en jaune-lors de la tâche «Imaginez jouer au tennis » (aire motrice supplémentaire) et-en vert-lors de la tâche «Imaginez visiter votre maison » (gyrus parahippocampique) chez un sujet sain. En bas : patient ne démontrant pas de signe de conscience au chevet, mais présentant une activation cérébrale similaire aux sujets témoins pour la tâche "Imaginez jouer au tennis ».

un rôle déterminant dans l'étude détaillée et rigoureuse de l'activité neuronale associée à la conscience.

De manière générale, l'électrogenèse des patients diagnostiqués en ECA est caractérisée par un ralentissement global constitué d'une augmentation des ondes lentes de type delta $[46,47]$. Certains chercheurs ont également mis en évidence une puissance delta plus élevée chez les patients en EV/ENR, comparativement aux patients en ECM [47]. De façon plus générale, il semble qu'une dominance des fréquences lentes, telles que delta, corrèlent avec de mauvais résultats cliniques, alors que les fréquences plus rapides, telles que alpha, corrèlent avec de bons résultats [48]. Une étude récente observant la connectivité cérébrale entre les différents sites d'électrodes suggère également que les patients en EV/ENR sont marqués par des réseaux moins bien connectés que les patients en ECM [47]. En somme, le niveau de connectivité semble lié à la sévérité du trouble de la conscience [49]. Dans le cas des patients atteints d'un syndrome locked-in, les résultats de l'électrogenèse corticale observés sont plus hétérogènes, traduisant par ailleurs l'incertitude et l'ambiguiité actuelles liées à la classification de leur état fonctionnel [50]. Aujourd'hui, un nombre croissant de chercheurs utilisent l'EEG pour mieux comprendre les troubles de la conscience et plusieurs paradigmes actifs, moyennant différentes composantes de l'activité électrique, ont été développés afin d'aider à la détection de signes de conscience [51-53].

En outre, certaines études ont mis en évidence la présence d'un rythme du sommeil comportemental (c'est-àdire la présence d'une période prolongée de fermeture des yeux et d'inactivité musculaire) chez les patients en EV/ENR ainsi que chez les patients en ECM. Néanmoins, seuls les patients en ECM semblent présenter un tracé électrophysiologique correspondant au cycle du sommeil (similaire aux tracés observés chez des sujets sains) [54].

Enfin, Piarulli et al. [55] ont montré que les patients en ECM présentent une entropie spectrale plus importante 
ainsi qu'une plus grande variabilité temporelle comparativement aux patients en EV/ENR. D'une manière générale, au niveau EEG, un faible niveau de conscience est caractérisé par des signaux stéréotypés donnant lieu à une entropie spectrale basse. A contrario, les signaux associés à la conscience présentent un degré de complexité élevée et, de facto, une entropie spectrale plus importante. Contrairement aux patients en EV/ENR chez lesquels aucune périodicité significative n'a été observée, les patients en ECM montrent des fluctuations de l'entropie spectrale avec des périodicités de 70 minutes. Ces auteurs suggèrent que cette périodicité, semblable à celle décrite chez des sujets sains éveillés, pourrait être liée aux fluctuations des capacités attentionnelles et de la vigilance chez les patients en ECM. Par ailleurs, la découverte d'une périodicité de 70 minutes dans l'entropie spectrale offre aux cliniciens une fenêtre temporelle intéressante pour l'évaluation clinique de la conscience [55].

\section{Stimulation magnétique transcrânienne (SMT) couplée à l'EEG}

D'autres méthodes ne requérant pas du patient qu'il comprenne ou suive des instructions ont été développées et sont désormais utilisées afin d'établir un diagnostic précis. Employée en clinique depuis près de 30 ans, la stimulation magnétique transcrânienne (SMT ; en anglais : «transcranial magnetic stimulation » ou TMS) est un outil non invasif permettant de stimuler des régions spécifiques du cerveau afin d'étudier leur intégrité ou de moduler leurs fonctions. Cette technique consiste à appliquer une impulsion magnétique brève qui va induire un courant électrique. Celui-ci va dépolariser les neurones se trouvant sous la région stimulée. En modulant les différents paramètres de stimulation (par exemple l'intensité, la fréquence et la durée de la stimulation), cet outil peut permettre d'activer, inhiber ou induire des changements d'activité à plus ou moins long terme dans une région corticale cible [56]. En outre, la SMT peut être couplée à l'EEG afin d'observer la réponse corticale suite aux stimulations déclenchées par la SMT (au lieu d'observer l'activité musculaire ou les réponses comportementales).

Depuis quelques années, un nombre croissant d'études utilisant la SMT couplée à l'EEG sont menées sur un large éventail de troubles afin de mieux comprendre les mécanismes neurophysiologiques du cerveau humain sain ou lésé. Grâce à cette technique de neuro-imagerie, des chercheurs ont récemment développé un indice de complexité perturbationnelle ( «Perturbational Complexity Index » en anglais ou $\mathrm{PCl}$; pouvant varier de 0 à 1) permettant de quantifier le niveau de conscience [57]. Il apparaît que cet indice permet, de façon fiable, de différencier les patients en EV/ENR et en ECM. Cet indice est élevé chez les patients en ECM (de même que chez les individus sains éveillés et les patients avec un syndrome locked-in) et faible chez les patients en EV/ENR (de même que chez les patients sous anesthésie générale), le seuil de conscience étant défini comme un indice supérieur à 0,3 . De façon générale, bien que d'autres recherches soient nécessaires pour confirmer et approfondir ces observations, les résultats présents suggèrent que la technique SMT-EEG permet de différencier à un niveau individuel les patients en EV/ERN et en ECM. D'un point de vue clinique, les techniques
d'EEG et de SMT, ainsi que le couplage des deux, constituent des méthodes intéressantes et prometteuses dans l'établissement d'un diagnostic et dans la détection des corrélats neuronaux de la conscience auprès de patients chez qui la communication est inexistante.

\section{Pronostic}

Le pronostic et la récupération des patients souffrant d'un ECA sont généralement considérés selon trois dimensions :

- la mortalité ;

- la récupération de la conscience ;

- la récupération fonctionnelle.

La récupération de la conscience correspond à la présence de signes clairs de conscience de soi et/ou de l'environnement. Plus précisément, ces signes peuvent se traduire par des réponses volontaires fluctuantes à une demande orale et/ou écrite, une poursuite visuelle ou encore des réponses émotionnelles contextualisées [9]. La récupération fonctionnelle est quant à elle caractérisée par l'émergence d'une communication fonctionnelle et/ou de l'utilisation fonctionnelle d'objets, ainsi que par la capacité à apprendre, à accomplir de nouvelles tâches et à participer à des activités personnelles, professionnelles ou récréatives $[8,9]$.

Le pronostic et la récupération des patients souffrant d'un ECA sont influencés par l'étiologie, la gravité de la lésion, la durée du coma, la durée de l'EV/ENR et l'âge du patient [8]. En effet, les chances de récupérer une conscience normale deviennent extrêmement faibles après 3 mois dans le cas d'une lésion cérébrale d'origine non traumatique et après 1 an dans le cas d'une lésion cérébrale d'origine traumatique. Plus précisément, approximativement $35 \%$ des patients diagnostiqués en EV/ENR à 3 mois après une lésion traumatique auront récupéré leur conscience 1 an après la lésion. Dans le cas d'une lésion non traumatique, ce pourcentage n'est plus que de $5 \%$. Les patients diagnostiqués en EV/ENR à 6 mois suite à une lésion traumatique présentent toujours $15 \%$ de chances de récupérer la conscience 1 an après la lésion, tandis que ces chances sont nulles chez les patients en EV/ENR dont la lésion est d'origine non traumatique [8].

Néanmoins, depuis l'étude menée par la Multi-Society Task Force en 1994, nous avons assisté, d'une part à l'amélioration des soins médicaux et de la prise en charge de patients en ECA, et d'autre part à l'introduction du diagnostic d'ECM dans la littérature [9]. Conséquemment, Giacino et al. [58] ont suggéré une révision des données relatives au pronostic de récupération chez ces patients. Les résultats d'études récentes, prenant en compte la distinction entre EV/ENR et ECM, ont notamment révélé qu'une minorité de patients ayant été diagnostiqués en EV/ENR ou en ECM à 1 an démontre une amélioration continue jusqu'à 5 ans après l'accident $[59,60]$. Par ailleurs, les patients souffrant d'un ECM d'origine traumatique présentent un pronostic de récupération plus favorable et montrent une moindre incapacité fonctionnelle comparativement aux patients en EV/ENR et aux patients en ECM d'origine non traumatique [61]. 


\section{Prise en charge thérapeutique}

La prise en charge quotidienne de la population de patients en EV/ENR ou en ECM est un défi pour tous les acteurs de la santé au vu de la dépendance complète concernant les activités de la vie quotidienne. Le temps de soin journalier est en effet estimé entre 4,5 et 7 heures (toilette, habillage, alimentation, soins infirmiers, séances de kinésithérapie, ergothérapie, orthophonie,...) [62]. Cette charge de travail pour l'équipe soignante, et particulièrement pour le personnel infirmier, est à-même d'engendrer un taux de surmenage non négligeable (18\%) [63]. Outre cette lourdeur de soins, les recommandations thérapeutiques concernant les patients en ECA sont relativement pauvres. Il n'y a, à l'heure actuelle, aucune recommandation factuelle concernant le traitement de ces patients $[64,65]$. Cependant, certaines études récentes ont démontré les potentiels effets bénéfiques de plusieurs interventions.

\section{Traitements pharmacologiques}

Il existe différentes options médicamenteuses pour tenter d'améliorer l'état de conscience des patients sévèrement cérébrolésés.

L'amantadine, un agent antiviral et dopaminergique, est le seul médicament dont l'efficacité a été prouvée dans un essai clinique randomisé et contrôlé chez 184 patients traumatiques au stade subaigu (4 à 16 semaines après l'accident). Dans cette étude, le groupe de patients recevant le traitement réel montrait une récupération significativement plus rapide par rapport au groupe recevant le traitement placebo, mesurée par la Disability Rating Scale [66] et la CRS-R [67]. Cette amélioration clinique induite par l'amantadine semble être liée à une augmentation du métabolisme au niveau des réseaux frontopariétaux interne et latéral, réseaux impliqués dans les processus de récupération de la conscience [68].

D'autres traitements semblent améliorer l'état de conscience de certains patients alors que leur indication première est toute autre. Le zolpidem, par exemple, est un agent non benzodiazépine à courte durée d'action, généralement utilisé en tant que sédatif pour lutter contre l'insomnie. Paradoxalement, il a déjà montré des effets spectaculaires sur les capacités cognitives de patients en EV/ENR (récupération de réponse à la commande et de communication fonctionnelle par exemple), mais ces effets disparaissent entièrement quelques heures après l'administration de la dose et nécessitent une administration quotidienne afin d'être maintenus [69]. Bien que parfois décrit comme un «médicament miracle », l'efficacité du zolpidem est très limitée puisque 5 à $10 \%$ des patients répondent positivement à ce traitement [70].

Un autre exemple est le baclofène, un agoniste des récepteurs $G A B A_{B}$, qui est prescrit dans les cas de spasticité sévère car il permet d'augmenter l'inhibition présynaptique au niveau spinal, et dès lors de réduire l'intensité des contractions musculaires réflexes [71]. Une augmentation des scores totaux à la CRS-R a pu été observée chez 5 patients en EV/ENR chronique à partir de 2 semaines après l'administration de baclofène par voie intrathécale [72]. Il est intéressant de noter qu'il ne s'agit pas uniquement d'une augmentation au niveau de la sous-échelle motrice par diminution éventuelle de la spasticité, mais également au niveau des sous-échelles qui concernent l'éveil, la fonction oromotrice et la communication. En effet, une autre étude a investigué les effets de la pompe à baclofène chez 6 patients en ECM ou en EV/ENR (en moyenne, 37 mois s'étaient écoulés entre la lésion cérébrale et la pose de la pompe à baclofène) et 2 parmi ceux-ci ont rempli par la suite les critères d'émergence de l'ECM [73]. Cette méthode est cependant hautement invasive et s'applique uniquement aux patients présentant une spasticité sévère et ne répondant pas aux traitements antispastiques oraux habituels. Notons également que ces études n'ont pas de comparateur placebo. Il est donc encore difficile de différencier l'effet réel du baclofène de l'évolution naturelle du patient. Des études contrôlées sur une plus large population de patients doivent encore confirmer le potentiel thérapeutique de ce médicament sur l'évolution cognitive et fonctionnelle des patients en ECA.

Ces options pharmacologiques sont régulièrement exploitées en phase aiguë (jusqu'à 3 mois après l'incident). Lorsque celles-ci sont épuisées, d'autres alternatives de traitement peuvent être envisagées.

\section{Revalidation clinique}

Ces traitements vont se baser sur le potentiel de plasticité cérébrale résiduelle après la lésion cérébrale. En effet, le cerveau adulte possède une certaine capacité de réorganisation synaptique permettant de compenser les fonctions atteintes [74]. Un moyen d'exploiter cette capacité est d'enrichir les stimuli environnementaux au niveau auditif, tactile et olfactif [75]. De nombreux programmes de stimulation sensorielle ou de musicothérapie sont utilisés dans les centres de rééducation, mais le manque de standardisation de ces interventions couplé à la faible taille des échantillons étudiés ne permet pas encore d'attribuer clairement des effets bénéfiques liés uniquement à cette approche. Les avancées récentes en IRM fonctionnelle et en EEG devraient permettre de pallier partiellement ce manque d'objectivité [76].

Un autre champ de traitement en plein essor pour les patients en ECA est la stimulation cérébrale invasive (stimulation cérébrale profonde) et non invasive (stimulation transcrânienne).

La stimulation cérébrale profonde (ou DBS pour « deep brain stimulation ») est une technique neurochirurgicale consistant à implanter des électrodes au niveau de régions cérébrales profondes. Cette méthode était initialement principalement utilisée pour traiter la maladie de Parkinson [77], mais ses applications se sont diversifiées (dystonie, douleur chronique et troubles obsessionnels compulsifs) [78]. Pour les patients en ECA, le thalamus est une cible de choix de cette technique étant donné qu'il a un rôle clé dans l'éveil et les fonctions cognitives élémentaires de mémoire de travail et de planification du mouvement [79]. Cette structure est d'ailleurs particulièrement atteinte chez les patients présentant des troubles de la conscience après une lésion cérébrale sévère [80]. La DBS est une technique hautement invasive, mais qui montre certains résultats prometteurs. À la suite d'une DBS au niveau de la formation réticulée, par exemple, 8 patients en EV/ENR chronique sur 21 ont récupéré une réponse à la commande 
et 4 patients en ECM sur 5 ont récupéré une communication fonctionnelle [81]. Cependant, cette étude ne comporte pas de comparateur placebo. Une autre étude, contrôlée cette fois, concerne le cas d'un patient en ECM chronique depuis 6 ans qui a reçu des sessions de DBS au niveau des noyaux intralaminaires et a immédiatement montré des verbalisations et une utilisation fonctionnelle d'objets, puis des réponses à la commande et une communication fonctionnelle [82]. L'utilisation de la DBS reste néanmoins peu accessible/peu utilisée/rarement pratiquée.

La stimulation électrique transcrânienne à courant continu (ou tDCS pour «transcranial direct current stimulation ») représente une alternative non invasive et moins lourde à déployer. Cette méthode de neuromodulation utilise un courant continu de faible intensité (de l'ordre du milliampère) appliqué directement sur le scalp au moyen d'électrodes, et ce afin d'augmenter l'excitabilité corticale de la région stimulée en abaissant le seuil de déclenchement des potentiels d'action neuronaux [83]. Une étude randomisée, contrôlée en double insu sur 55 patients en ECA, a montré une augmentation significative du score à la CRS-R après une seule session de tDCS qu'on ne retrouve pas après une stimulation placebo [84]. Dans cet échantillon, le sousgroupe de patients en ECM montre une augmentation du score proportionnellement plus importante que les patients en EV/ENR. Cependant, les effets ne sont que transitoires et nécessitent des stimulations répétées quotidiennement afin d'être maintenus. Cette technique ne présentant aucun effet secondaire sévère et étant facile à intégrer dans les programmes de rééducation par sa simplicité d'utilisation, elle représente un excellent candidat dans l'arsenal thérapeutique pour les patients en ECA.

\section{Soins de confort}

Parallèlement à ces traitements actifs, les soins de confort ne sont certainement pas à négliger chez les patients en ECA, d'autant plus que ceux-ci ne sont que très rarement à-même d'exprimer leurs maux. De plus, il a été démontré que les patients en ECM peuvent ressentir de la douleur [85].

Cliniquement, il est difficile d'adapter de manière adéquate un traitement chez les patients non communicants, puisqu'il est impossible d'obtenir un feed-back de leur part sur leur ressenti. Des études ont montré que la «Nociception Coma Scale-Revised » (NCS-R), évaluant la douleur et la nociception des patients en ECA, est un outil adéquat permettant d'apprécier les réponses des patients à des stimuli douloureux $[86,87]$. La NCS-R comprend trois sous-échelles évaluant les réponses motrices, verbales et faciales du patient. Elle peut être appliquée lors d'un soin potentiellement douloureux. Un seuil de 4 ou plus suggère la présence de douleurs. Une étude utilisant le PET-scan a montré une corrélation positive entre les scores totaux de la NCS-R et le métabolisme cérébral de la partie postérieure du cortex cingulaire antérieur, une région connue pour son implication dans les processus cognitifs et affectifs de la gestion de la douleur [88]. Ces résultats confirment l'hypothèse selon laquelle la NCS-R est liée au traitement cortical de la douleur. Cette échelle pourrait ainsi constituer un outil comportemental approprié pour le contrôle de la douleur chez les patients non communicants.
La particularité de cette population de patients, en plus de l'incapacité à communiquer, est l'étendue des lésions au niveau du système nerveux central qui mène, dans $89 \%$ des cas [89], à l'apparition de la spasticité. Plusieurs options permettent de lutter contre cette spasticité :

- chirurgie orthopédique (ténotomie, DREZotomie, neurotomie) ;

- médication (baclofène oral ou intrathécal) ;

- kinésithérapie (étirements, postures, mobilisations) ;

- traitements passifs (plâtres, orthèses, attelles).

L'approche pluridisciplinaire est généralement privilégiée. Un adjuvant à cette approche pour le membre supérieur est l'utilisation d'attelles souples en mousse placées au creux de la main. Ces attelles permettent une ouverture de main prolongée tout en évitant les troubles circulatoires et les plaies généralement observés après l'utilisation d'attelles rigides. Une étude a montré une diminution significative de la spasticité (mesurée par l'échelle d'Ashworth) au niveau des muscles de la main dans un échantillon de 17 patients spastiques à la suite du port de ces attelles [90].

Ces soins de confort peuvent permettre au patient de montrer certains signes de conscience parfois occultés par la spasticité ou la douleur et jouent donc également un rôle essentiel dans le diagnostic. La balance entre traitements actifs et passifs est à déterminer au cas par cas, en fonction du stade de la lésion, des capacités du patient et des éventuelles attentes de celui-ci et de ses proches.

\section{Éthique et fin de vie}

La prise en charge de patients présentant une altération de la conscience demeure un véritable défi. Néanmoins, le développement des techniques de neuro-imagerie nous permet désormais d'affiner leur diagnostic. Une fois ce diagnostic correctement posé, différentes questions surgiront inévitablement quant à la qualité de vie de ces patients : combien de temps faudra-t-il attendre afin qu'il/elle récupère une conscience? Qu'est-ce qu'une récupération significative ? L'émergence et le développement des connaissances sur les différents états de conscience vont, d'une part, accroître les attentes de certaines familles et, d'autre part, apporter une déception sans précédent à d'autres [91]. Comme le soulignent à juste titre Fins et al. [91], les troubles de la conscience ont un impact non négligeable sur l'entourage des patients sévèrement cérébrolésés qui sera inévitablement impliqué dans les décisions de prises en charge et de fin de vie.

L'établissement d'un pronostic, la conception d'un plan de soin adapté et la justesse des informations dispensées au personnel soignant dépendront de la précision du diagnostic établi. Malheureusement, comme cela a été évoqué par Giacino et al. [58], une erreur diagnostique peut entraîner une gestion médicale inappropriée, comme la négligence de symptômes douloureux, ou encore un retrait prématuré des soins de maintien des fonctions vitales [64]. Or, il a été démontré que 30 à $40 \%$ des patients ayant été diagnostiqués en EV/ENR présentent en fait des signes de conscience [12]. 


\section{Conclusion}

Certains patients sont susceptibles de demeurer dans un ECA durant de nombreuses années. Dans cette perspective, l'amélioration des diverses techniques d'évaluation et de prises en charge des troubles de la conscience apparaît essentielle. Dans cette revue, nous avons décrit différentes méthodes de diagnostic, de l'échelle CRS-R aux techniques d'imagerie médicale comprenant l'IRM, la TEP ou encore la SMT-EEG. En fonction du diagnostic établi, ainsi que de l'étiologie, un certain pronostic peut être avancé. Celuici revêt une importance éthique capitale, particulièrement lorsque la fin de vie d'un patient doit être évoquée. Dans le cas contraire, la prise en charge pharmacologique et la revalidation clinique, via différentes stimulations basées sur la plasticité cérébrale résiduelle, peuvent être envisagées. Si l'objectif principal de cette revue était de rendre compte de l'avancement de la recherche dans ce domaine relativement récent, de nombreuses techniques diagnostiques et thérapeutiques seront encore développées dans le futur.

\section{Remerciements}

Ce projet a été financé par l'université et l'hôpital universitaire de Liège, le Fonds belge de la recherche scientifique (FRS-FNRS), la Commission européenne, Luminous, Human Brain Project, Center-TBI, la James McDonnell Foundation, la European Space Agency, Belspo, la Fondazione Europea di Ricerca Biomedica, la BIAL Foundation, le programme Actions de recherche concertées (ARC) de la Fédération Wallonie-Bruxelles, ainsi que la Mind Science Foundation.

\section{Déclaration de liens d'intérêts}

Les auteurs déclarent ne pas avoir de liens d'intérêts.

\section{Références}

[1] Laureys S. The neural correlate of (un)awareness: lessons from the vegetative state. Trends Cogn Sci 2005;9:556-9.

[2] Laureys S, Boly M. The changing spectrum of coma. Nat Clin Pr Neurol 2008;4:544-6.

[3] Schnakers C, Majerus S. Évaluation comportementale et diagnostic des états de conscience altérée. In: Schnakers C, Laureys S, editors. Coma et états de conscience altérée. Vienna: Springer; 2011. p. 3-15.

[4] Gosseries O, Zasler ND, Laureys S. Recent advances in disorders of consciousness: focus on the diagnosis. Brain Inj 2014;28:1141-50.

[5] Laureys S, Owen AM, Schiff ND. Brain function in coma, vegetative state and related disorders. Lancet Neurol 2004;3:537-46.

[6] Laureys S, Celesia GG, Cohadon F, et al. Unresponsive wakefulness syndrome: a new name for the vegetative state or apallic syndrome. BMC Med 2010;8:68.

[7] The Quality Standards Subcommittee of the American Academy of Neurology. Practice parameters for determining brain death in adults (summary statement). Neurology 1995;45:1012-4.

[8] The Multy-Society Task Force on PVS. Medical aspects of the persistent vegetative state (2). New Engl J Med 1994;330:1499-508.

[9] Giacino JT, Ashwal S, Childs N, et al. The minimally conscious state. Neurology 2002;58:349-53.
[10] Bruno M-A, Majerus S, Boly M, et al. Functional neuroanatomy underlying the clinical subcategorization of minimally conscious state patients. J Neurol 2012;259:1087-98.

[11] Leonardi M, Sattin D, Raggi A. An Italian population study on 600 persons in vegetative state and minimally conscious state. Brain Inj 2013;27:473-84.

[12] Schnakers C, Vanhaudenhuyse A, Giacino J, et al. Diagnostic accuracy of the vegetative and minimally conscious state: clinical consensus versus standardized neurobehavioral assessment. BMC Neurol 2009;9:35.

[13] Stender J, Gosseries O, Bruno MA, et al. Diagnostic precision of PET imaging and functional MRI in disorders of consciousness: a clinical validation study. Lancet 2014;384:514-22.

[14] Teasdale G, Jennett B. Assessment of coma and impaired consciousness. Lancet 1974;304:81-4.

[15] Laureys S, Bodart O, Gosseries O. The Glasgow Coma Scale: time for critical reappraisal? Lancet Neurol 2014;13:755-7.

[16] Seel RT, Sherer M, Whyte J, et al. Assessment scales for disorders of consciousness: evidence-based recommendations for clinical practice and research. Arch Phys Med Rehabil 2010;91:1795-813.

[17] Giacino JT, Kalmar K, Whyte J, The JFK. Coma Recovery ScaleRevised: measurement characteristics and diagnostic utility. Arch Phys Med Rehabil 2004;85:2020-9.

[18] Bodien YG, Carlowicz CA, Chatelle C, et al. Sensitivity and specificity of the coma recovery scale-revised total score in detection of conscious awareness. Arch Phys Med Rehabil 2016;97:490-2.

[19] Estraneo A, Moretta P, Cardinale V, et al. A multicentre study of intentional behavioral responses measured using the Coma Recovery Scale - revised in patients with minimally conscious state. Clin Rehabil 2014;29:5-10.

[20] Bagnato S, Boccagni C, Sant'Angelo A, et al. Longitudinal assessment of clinical signs of recovery in patients with unresponsive wakefulness syndrome after traumatic or nontraumatic brain injury. J Neurotrauma 2016;33:1-5.

[21] Vanhaudenhuyse A, Schnakers C, Bredart S, et al. Assessment of visual pursuit in post-comatose states: use a mirror. J Neurol Neurosurg Psychiatry 2008;79:223-33.

[22] Thonnard M, Wannez S, Keen S, et al. Detection of visual pursuit in patients in minimally conscious state: a matter of stimuli and visual plane? Brain Inj 2014;28:1164-70.

[23] Gosseries O, Zasler ND, Laureys S. Recent advances in disorders of consciousness: focus on the diagnosis. Brain Inj 2014;28:1362-2301.

[24] Di H, Nie Y, Hu X, et al. Assessment of visual fixation in vegetative and minimally conscious states. BMC Neurol 2014;14: 147.

[25] Wannez S, Gosseries O, Azzolini D, et al. Prevalence of comarecovery scale-revised signs of consciousness in patients in minimally conscious state. Neuropsychol Rehabil 2017:1-10.

[26] Lovstad M, Froslie KF, Giacino JT, et al. Reliability and diagnostic characteristics of the JFK coma recovery scale-revised: exploring the influence of rater's level of experience. J Head Trauma Rehabil 2010;25:349-56.

[27] Godbolt AK, Stenson S, Winberg M, et al. Disorders of consciousness: preliminary data supports added value of extended behavioral assessment. Brain Inj 2012;26:188-93.

[28] Sattin D, Giovannetti AM, Ciaraffa F, et al. Assessment of patients with disorder of consciousness: do different Coma Recovery Scale scoring correlate with different settings? J Neurol 2014;261:2378-86.

[29] Bruno M-A, Ledoux D, Vanhaudenhuyse A, et al. Pronostic des patients récupérant du coma. In: Schnakers C, Laureys S, editors. Coma et états de conscienc altérée. Vienna: Springer; 2011. p. 17-30.

[30] Demertzi A, Schnakers C, Ledoux D, et al. Different beliefs about pain perception in the vegetative and minimally 
conscious states: a European survey of medical and paramedical professionals. Prog Brain Res 2009;177:329-38.

[31] Demertzi A, Ledoux D, Bruno MA, et al. Attitudes towards endof-life issues in disorders of consciousness: a European survey. J Neurol 2011;258:1058-65.

[32] Giacino JT, Hirsch J, Schiff N, et al. Functional neuroimaging applications for assessment and rehabilitation planning in patients with disorders of consciousness. Arch Phys Med Rehabil 2006;87:67-76

[33] Majerus S, Bruno MA, Schnakers C, et al. The problem of aphasia in the assessment of consciousness in brain-damaged patients. Prog Brain Res 2009;177:49-61.

[34] Kirsch M, Wannez S, Thibaut A, et al. Positron emission tomography: basic principles, new applications and studies under anesthesia. Handb Clin Neurol 2016;54:109-28.

[35] Stender J, Kupers R, Rodell A, et al. Quantitative rates of brain glucose metabolism distinguish minimally conscious from vegetative state patients. J Cereb Blood Flow Metab 2015;35:58-65.

[36] Laureys S, Lemaire C, Maquet P, et al. Cerebral metabolism during vegetative state and after recovery to consciousness. J Neurol Neurosurg Psychiatry 1999;67:121-33.

[37] Laureys S, Antoine S, Boly M, et al. Brain function in the vegetative state. Acta Neurol Belg 2002;102:177-85.

[38] Vanhaudenhuyse A, Demertzi A, Schabus M, et al. Two distinct neuronal networks mediate the awareness of environment and of self. J Cogn Neurosci 2011;23:570-8.

[39] Guldenmund P, Soddu A, Baquero K, et al. Structural brain injury in patients with disorders of consciousness: a voxelbased morphometry study. Brain Inj 2016;30:343-52.

[40] Zheng ZS, Reggente N, Lutkenhoff E, et al. Disentangling disorders of consciousness: insights from diffusion tensor imaging and machine learning. Hum Brain Mapp 2016;38:431-43.

[41] Owen AM. Detecting awareness in the vegetative state. Science 2006;313:1402.

[42] Schiff ND, Rodriguez-Moreno D, Kamal A, et al. fMRI reveals large-scale network activation in minimally conscious patients. Neurology 2005;64:514-23.

[43] Heine L, Castro M, Martial C, et al. Exploration of functional connectivity during preferred music stimulation in patients with disorders of consciousness. Front Psychol 2015;6:1-11.

[44] Demertzi A, Antonopoulos G, Heine L, et al. Intrinsic functional connectivity differentiates minimally conscious from unresponsive patients. Brain 2015;138:2619-31.

[45] Di Perri C, Bahri MA, Amico E, et al. Neural correlates of consciousness in patients who have emerged from a minimally conscious state: a cross-sectional multimodal imaging study. Lancet Neurol 2016;15:830-42.

[46] Leon-carrion J, Martin-rodriguez JF, Damas-lopez J, et al. Brain function in the minimally conscious state: a quantitative neurophysiological study. Clin Neurophysiol 2008;119:1506-14.

[47] Lehembre R, Gosseries O, Lugo Z, et al. Electrophysiological investigations of brain function in coma, vegetative and minimally conscious patients. Arch Ital Biol 2012;150:122-39.

[48] Bagnato S, Istituto F, Giglio G, et al. EEG predictors of outcome in patients with disorders of consciousness admitted for intensive rehabilitation admitted for intensive rehabilitation. Clin Neurophysiol 2015;126:959-66.

[49] Pollonini L, Pophale S, Situ N, et al. Information communication networks in severe traumatic brain injury. Brain Topogr 2010;23:221-6.

[50] Leon-Carrion J, Eeckhout P, van, Dominguez-Morales M, del R. Review of subject: the locked-in syndrome: a syndrome looking for a therapy. Brain Inj 2002;16:555-69.

[51] Bruno MA, Soddu A, Demertzi A, et al. Disorders of consciousness: moving from passive to resting state and active paradigms. Cogn Neurosci 2010;1:193-203.
[52] Lulé D, Noirhomme Q, Kleih SC, et al. Clinical neurophysiology probing command following in patients with disorders of consciousness using a brain - computer interface. Clin Neurophysiol 2013;124:101-6.

[53] Cruse D, Chennu S, Chatelle C, et al. Bedside detection of awareness in the vegetative state: a cohort study. Lancet 2011;378:2088-94.

[54] Landsness E, Bruno MA, Noirhomme Q, et al. Electrophysiological correlates of behavioural changes in vigilance in vegetative state and minimally conscious state. Brain 2011;134: $2222-32$.

[55] Piarulli A, Bergamasco M, Thibaut A, et al. EEG ultradian rhythmicity differences in disorders of consciousness during wakefulness. J Neurol 2016;263:1746-60.

[56] Wassermann EM, Lisanby SH. Therapeutic application of repetitive transcranial magnetic stimulation: a review. Clin Neurophysiol 2001;112:1367-77.

[57] Casali AG, Gosseries O, Rosanova M, et al. A Theoretically Based index of consciousness independent of sensory processing and behavior. Sci Transl Med 2013;5:1-10.

[58] Giacino JT, Katz D, Whyte J. Neurorehabilitation in disorders of consciousness. Semin Neurol 2013;33:142-56.

[59] Katz DI, Polyak M, Coughlan D, et al. Natural history of recovery from brain injury after prolonged disorders of consciousness: outcome of patients admitted to inpatient rehabilitation with 1-4 year follow-up. Prog Brain Res 2009;177:73-88.

[60] Nakase-Richardson R, Whyte J, Giacino JT, et al. Longitudinal outcome of patients with disordered consciousness in the NIDRR TBI model systems programs. J Neurotrauma 2012;29:59-65.

[61] Lammi MH, Smith VH, Tate RL, et al. The minimally conscious state and recovery potential: a follow-up study 2 to 5 years after traumatic brain injury. Arch Phys Med Rehabil 2005;86:746-54.

[62] Saoût V, Ombredane MP, Mouillie JM, et al. Patients in a permanent vegetative state or minimally conscious state in the Maine-et-Loire county of France: a cross-sectional, descriptive study. Ann Phys Rehabil Med 2010;53:96-104.

[63] Gosseries O, Demertzi A, Ledoux D, et al. Burnout in healthcare workers managing chronic patients with disorders of consciousness. Brain Inj 2012;26:1493-9.

[64] Giacino JT, Fins JJ, Laureys S, et al. Disorders of consciousness after acquired brain injury: the state of the science. Nat Rev Neurol 2014;10:99-114.

[65] Bernat JL. Chronic disorders of consciousness. Lancet 2006;367:1181-92.

[66] Rappaport M, Hall KM, Hopkins K, et al. Disability rating scale for severe head trauma: coma to community. Arch Phys Med Rehabil 1982;63:118-23.

[67] Giacino JT, Whyte J, Bagiella E, et al. Placebo-controlled trial of amantadine for severe traumatic brain injury. N Engl J Med 2012;366:819-26.

[68] Schnakers C, Hustinx R, Vandewalle G, et al. Measuring the effect of amantadine in chronic anoxic minimally conscious state. J Neurol Neurosurg Psychiatry 2008;79:225-7.

[69] Clauss R, Nel W. Drug induced arousal from the permanent vegetative state. Neuro Rehabilitation 2006;21:23-8.

[70] Gosseries O, Charland-Verville V, Thonnard M, et al. Amantadine, apomorphine and zolpidem in the treatment of disorders of consciousness. Curr Pharm Des 2014;20: 4167-84.

[71] Leong B. The vegetative and minimally conscious states in children: spasticity, muscle contracture and issues for physiotherapy treatment. Brain Inj 2002;16:217-30.

[72] Sarà M, Pistoia F, Mura E, et al. Intrathecal baclofen in patients with persistent vegetative state: 2 hypotheses. YAPMR 2009;90:1245-9. 
[73] Margetis K, Korfias SI, Gatzonis S, et al. Intrathecal baclofen associated with improvement of consciousness disorders in spasticity patients. Neuromodulation 2013;17: 699-704.

[74] Hummel FC, Cohen LG. Drivers of brain plasticity. Curr Opin Neurol 2005;18:667-74.

[75] Nithianantharajah J, Hannan AJ. Enriched environments, experience-dependent plasticity and disorders of the nervous system. Nat Rev Neurosci 2006;7:697-709.

[76] Schnakers C, Magee WL, Harris B. Sensory stimulation and music therapy programs for treating disorders of consciousness. Front Psychol 2016;7:1-6.

[77] Dormont D, Seidenwurm D, Galanaud D, et al. Neuroimaging and deep brain stimulation. Am J Neuroradiol 2010;31: 15-23.

[78] Thibaut A, Laureys S. Brain stimulation in patients with disorders of consciousness. Princ Pract Clin Res 2015;1: 65-70.

[79] Schiff ND, Giacino JT, Fins JJ. Deep brain stimulation, neuroethics, and the minimally conscious state: moving beyond proof of principle. Arch Neurol 2009;66:697-702.

[80] Schiff ND. Central thalamic contributions to arousal regulation and neurological disorders of consciousness. Ann N Y Acad Sci 2008;1129:105-18.

[81] Yamamoto T, Kobayashi K, Kasai M, et al. DBS therapy for the vegetative state and minimally conscious state. In: von Wild $\mathrm{KRH}$, editor. Re-engineering of the damaged brain and spinal cord. New-York: Springer; 2005. p. 101-4.
[82] Schiff ND, Giacino JT, Kalmar K, et al. Behavioral improvements with thalamic stimulation after severe traumatic brain injury. Nature 2007;448:600-3.

[83] Stagg CJ, Nitsche MA. Physiological basis of transcranial direct current stimulation. Neuroscientist 2011;17:37-53.

[84] Thibaut A, Bruno MA, Demertzi A, et al. tDCS in patients with disorders of consciousness. Neurology 2014;82:1112-8.

[85] Boly M, Faymonville ME, Schnakers C, et al. Perception of pain in the minimally conscious state with PET activation: an observational study. Lancet Neurol 2008;7:1013-20.

[86] Schnakers C, Chatelle C, Vanhaudenhuyse A, et al. The Nociception Coma Scale: a new tool to assess nociception in disorders of consciousness. Pain 2010;148:215-9.

[87] Chatelle C, Majerus S, Whyte J, et al. A sensitive scale to assess nociceptive pain in patients with disorders of consciousness. $J$ Neurol Neurosurg Psychiatry 2012;83:1233-7.

[88] Chatelle C, Thibaut A, Whyte J, et al. Pain issues in disorders of consciousness. Brain Inj 2014;28:1202-8.

[89] Thibaut A, Chatelle C, Wannez S, et al. Spasticity in disorders of consciousness: a behavioral study. Eur J Phys Rehabil Med 2014;51:389-97.

[90] Thibaut A, Deltombe T, Wannez S, et al. Impact of soft splints on upper limb spasticity in chronic patients with disorders of consciousness: a randomized, single-blind, controlled trial. Brain Inj 2015;29:830-6.

[91] Fins JJ, Illes J, Bernat JL, et al. Neuroimaging and disorders of consciousness: envisioning an ethical research agenda. Am J Bioeth 2008;8:3-12. 